Research Article

\title{
Transcriptional Response of Multidrug-Resistant Klebsiella pneumoniae Clinical Isolates to Ciprofloxacin Stress
}

\author{
Roman Farooq Alvi, ${ }^{1}$ Bilal Aslam (D), ${ }^{1}$ Muhammad Hidayat Rasool $\left(\mathbb{D},{ }^{1}\right.$ Saima Muzammil (iD, \\ Abu Baker Siddique $\mathbb{D}^{1},{ }^{1}$ Nafeesa Yasmeen, ${ }^{2}$ Mohsin Khurshid ${ }^{D},{ }^{1}$ Noreen Sarwar, \\ Ahmad Almatroudi $\left(\mathbb{D},{ }^{4}\right.$ Riaz Hussain, ${ }^{5}$ and Zulqarnain Baloch $\mathbb{D}^{6}$ \\ ${ }^{1}$ Department of Microbiology, Government College University Faisalabad, Faisalabad, Pakistan \\ ${ }^{2}$ College of Veterinary Medicine, South China Agricultural University, Guangzhou 610642, China \\ ${ }^{3}$ Institute of Microbiology, University of Veterinary and Animal Sciences, Lahore, Pakistan \\ ${ }^{4}$ Department of Medical Laboratories, College of Applied Medical Sciences, Qassim University, Buraydah, Saudi Arabia \\ ${ }^{5}$ University College of Veterinary and Animal Sciences, The Islamia University of Bahawalpur, Bahawalpur, Pakistan \\ ${ }^{6}$ Faculty of Life Science and Technology, Kunming University of Science and Technology, Kunming, Yunnan 650500, China
}

Correspondence should be addressed to Bilal Aslam; drbilalaslam@gcuf.edu.pk and Ahmad Almatroudi; aamtrody@qu.edu.sa

Received 16 February 2021; Accepted 1 November 2021; Published 28 November 2021

Academic Editor: Mian Muhammad Awais

Copyright (c) 2021 Roman Farooq Alvi et al. This is an open access article distributed under the Creative Commons Attribution License, which permits unrestricted use, distribution, and reproduction in any medium, provided the original work is properly cited.

\begin{abstract}
Background. The term "persisters" refers to a small bacterial population that persists during treatment with high antibiotic concentration or dose in the absence of genetic resistance. The present study was designed to investigate the transcriptional response in indigenous Klebsiella pneumoniae under the ciprofloxacin stress. Methods. Isolation and identification of K. pneumoniae were carried out through standard microbiological protocols. The characterization of quinolone resistance was performed by estimating the quinolone susceptibility testing, MIC estimation, and detecting the QRDR and PMQR. Transcriptional response of the isolates to ciprofloxacin was determined using qPCR. Results. Among 34 isolates, 23 (67\%) were resistant to ciprofloxacin. Both QRDR ( $g y r A$ and $g y r B$ ) and PMQR ( $q n r A$, $q n r B$, and $q n r S$ ) were detected in the isolates, and all were found resistant to ciprofloxacin. The mRNA levels of both mutS and euTu under the influence of ciprofloxacin were significantly increased. On ciprofloxacin exposure, the mRNA levels of the DNA damage response element (mutS) were raised in a time-dependent fashion. K. pneumoniae showed high-level resistance to ciprofloxacin in the presence of mutations in QRDR and PMQR genes. Conclusion. The transcriptional response revealed the upregulation of DNA repair and protein folding elements $(m u t S$ and $e u T u$ ) in ciprofloxacin stress and delayed cell division. The ciprofloxacin was found to trigger various stress responses in a time- and concentration-dependent manner.
\end{abstract}

\section{Introduction}

The term "persisters" refers to a small bacteria population that persists during treatment with high antibiotics concentration or dose in the absence of genetic resistance [1]. There are different host and bacterial potential factors which help the pathogens to evade the host immune system and persist within the host cells. Among them, sublethal antibiotic concentration is one of the potential factors associated with the development of persistent resistant bacterial strains
[2]. Klebsiella pneumoniae is a Gram-negative bacterium of the Enterobacteriaceae family and associated with a variety of infections in humans and animals. Among Gram-negative bacterial infections, almost one-third of the infections are caused by Klebsiella pneumoniae [3]. The emergence of multidrug-resistant (MDR) bacterial strains is a global health concern and very difficult to treat [4]. Sublethal dosage and inadequate regime of antibiotics steer the evolution of resistant superbugs. It has already been established that sublethal concentration of bactericidal antibiotics 
triggers the development of MDR, tolerant, or persistent Klebsiella pneumoniae [5].

Generally, bacteria possess a strong defense mechanism against the antibiotics, which triggers the induction of various adaptive responses and reactions at various stages of the cell cycle. Usually, these adaptive responses are known to be the stress response of the bacteria. The particular stress response is incorporated into a flexible adaptive network which is relative to environmental indicators or signals, and it can increase the antibiotic resistance and may maintain the bacterial viability which may ultimately resume the growth of bacteria once again in the optimum environmental conditions [6]. The concentration of antibiotics may be a reason which triggers the global stress response of the bacteria, or it may also generate a target-specific response [7]. Antibiotics themselves may be considered as stressors because they not only interfere with various cellular processes but the evolution of some stress responses in bacteria due to antibiotics has also been reported, which is linked with the emergence of antibiotic resistance $[8,9]$.

Ciprofloxacin is a broad-spectrum fluoroquinolone antibiotic that hinders the replication of bacterial DNA by impeding DNA gyrase and topoisomerase. Ciprofloxacin is extensively prescribed in the clinical settings of Pakistan to treat various bacterial infections such as typhoid fever and respiratory tract infection [10]. Recently, we have reported the molecular mechanism of resistance against ciprofloxacin in indigenous Klebsiella pneumoniae isolates [11]. The present study is designed to investigate the transcriptional response in indigenous Klebsiella pneumoniae under the stress of ciprofloxacin, which ultimately is helpful to find out the link between ciprofloxacin stress and its resistance in indigenous Klebsiella pneumoniae isolates.

\section{Methods}

2.1. Isolation and Identification of Klebsiella pneumoniae. The cross-sectional study was performed at the Department of Microbiology, Government College University, Faisalabad, Pakistan. The protocol used in this study was in accordance with the Declaration of Helsinki and was approved by the Ethics Review Committee (ERC) at Government College University, Faisalabad, Pakistan, No: ERC/GCUF/137-18.

All isolates originated from blood cultures were collected from different reference hospitals of Lahore and Faisalabad, the two metropolitan cities of Pakistan. A total of 200 blood samples were collected from patients suspected of bloodstream infections according to standard indications and methods as described elsewhere [12]. A representative selection of the isolates (10\%) was sent to the biomedical Research Center, Northwest Minzu University. The isolation of $K$. pneumoniae was carried out from various clinical samples $(N=200)$ by streaking on Hi-Crome KlebsiellaSelective Agar Base M 1573 (HIMEDIA ${ }^{\circledR}$ ). Standard microbiological procedures were adopted for morphological, cultural, and biochemical profiling of the isolates using the API 20E kit (bioMérieux, France). Additionally, the molecular identification of the isolates was performed through PCR using specific primers against 16S rDNA (Table 1).
TABLE 1: Sequence details of various primers used in the study.

\begin{tabular}{|c|c|}
\hline Gene name & Primer sequences \\
\hline $16 S$ rDNA & $\begin{array}{l}\text { AGAGTTTGATCTGGCTCAG } \\
\text { AAGGAGGTGWTCCACC }\end{array}$ \\
\hline gyrA & $\begin{array}{l}\text { GGTACACCGTCGCGTACTTT } \\
\text { TCGATGGAACCGAAGTTACC }\end{array}$ \\
\hline gyrB & $\begin{array}{c}\text { CTCCGTCTCCGTACAGGATGAC } \\
\text { TGTGATAGCGCAGTTTATCC }\end{array}$ \\
\hline$q n r A$ & $\begin{array}{l}\text { ATTTCTCACGCCAGGATTTG } \\
\text { GATCGGCAAAGGTTAGGTCA }\end{array}$ \\
\hline$q n r B$ & $\begin{array}{l}\text { GATCGTGAAAGCCAGAAAGG } \\
\text { ACGATGCCTGGTAGTTGTCC }\end{array}$ \\
\hline$q n r S$ & $\begin{array}{l}\text { ACGACATTCGTCAACTGCAA } \\
\text { TAAATTGGCACCCTGTAGGC }\end{array}$ \\
\hline $\operatorname{lex} \mathrm{A}$ & $\begin{array}{l}\text { TCAGTGGGATGTCGATGAAA } \\
\text { GCCAGACCCTCAATGGTAAA }\end{array}$ \\
\hline$e u-\mathrm{Tu}$ & $\begin{array}{l}\text { CAGGTAGGCGTTCCGTACAT } \\
\text { CCAGCCAGTTCGATGATTTT }\end{array}$ \\
\hline muts & $\begin{array}{l}\text { GCTGTGGGAATTTGAAATCG } \\
\text { AGGTACGCTGGGTGTCTTTG }\end{array}$ \\
\hline$d n a \mathrm{~K}$ & $\begin{array}{l}\text { CCAGGACGAAGAAGTTCAGC } \\
\text { GAAGTAAGCCGGTACGGTGA }\end{array}$ \\
\hline$f t s Z$ & $\begin{array}{l}\text { ATGTCAGAAATGGGCTACGC } \\
\text { ACGGTTTCGAACTCATCCAG }\end{array}$ \\
\hline$c l p \mathrm{~B}$ & $\begin{array}{l}\text { GTACTGCAGCGTCGTACCAA } \\
\text { GAATGACGTTGCCTTCCTGT }\end{array}$ \\
\hline
\end{tabular}

2.2. Quinolone Susceptibility Testing (QST). Quinolone susceptibility testing of all the isolates was initially conducted by an E-test (bioMérieux, France) followed by the broth dilution method. Interpretation of results was performed according to the CLSI guideline 2018 [13].

2.3. Molecular Characterization of Quinolone Resistance Determinants. Quinolone resistance-determining regions (QRDRs), i.e., gyrA and parC genes, were identified through PCR, followed by sequencing and mutation detection. Additionally, plasmid-mediated quinolone resistance (PMQR) was also investigated through PCR by detecting various PMQR determinants which include $q n r A, q n r B$, and $q n r S$ genes with the help of specific primers (Table 1). All the PCR products were sent to Macrogen Korea for sequencing. Sequence analysis was conducted by software MEGA7. The database named "qnr Numbering and Sequence" which is available online (https://www.lahey.org/qnrstudies) was used for the analysis of PMQR [14]. Lastly, downstream to the BLAST analysis, all the obtained sequences were aligned and submitted to the NCBI (GenBank) for the allotment of accession numbers.

2.4. Primer Designing for Transcriptional Studies. The wholegenome sequence of $K$. pneumoniae was obtained from the NCBI for the synthesis of real-time PCR primers. Primers were designed using Primer3web version 4.0.0 (https:// primers.ut.ee/). The chemically synthesized primers were 
evaluated by conducting PCR from the genomic DNA of known K. pneumonia.

\subsection{Bacterial Growth Curve and Ciprofloxacin Persistence} Assay. To get the growth patterns of the selected K. pneumoniae clinical isolate, the growth curve was built by measuring absorbance as a function of bacterial growth. The isolate was cultivated in liquid nutrient broth (SigmaAldrich ${ }^{\circledR}$ USA) under the ciprofloxacin stress, and results were compared with bacteria growing under standard culture conditions.

2.6. Synthesis of Complementary DNA. RNA extraction was performed using a Trizol reagent (Invitrogen, Carlsbad, CA, USA) following the manufacturer's directions. Briefly, overnight-grown culture was subjected to centrifuge to get the pellet. The bacterial pellet was resuspended in cold PBS and TRIzol was added @ $0.4 \mathrm{ml} / 1 \times 10^{7}$ and incubated for $10 \mathrm{~min}$. Afterward, $100 \mu \mathrm{l}$ chloroform was added, and the cells were incubated for $5 \mathrm{~min}$. Centrifugation of the sample was carried out at $12,000 \times \mathrm{g}\left(4^{\circ} \mathrm{C}\right)$ for $15 \mathrm{~min}$, and the aqueous phase was taken up as RNA [15].

Reverse transcriptase (Revert Aid ${ }^{\mathrm{TM}} \mathrm{MMuLV}$, Fermentas) according to the manufacturer's instructions was used, and for PCR reaction mixture consisting of $4 \mu \mathrm{g}$ cellular RNA, $1.0 \mathrm{mM}$ dNTPs, $5 \mu \mathrm{M}$ random hexamers, and $1 \mathrm{X} \mathrm{M}-\mathrm{MLuV}$ buffer solution were used. The PCR reaction mixture was placed in a thermal cycler at $65^{\circ} \mathrm{C}$ for 6 minutes, $4^{\circ} \mathrm{C}$ for 5 minutes, $25^{\circ} \mathrm{C}$ for 12 minutes, and $42^{\circ} \mathrm{C}$ for 3 minutes followed by the addition of MMuLV $(200 \mathrm{U} / \mu \mathrm{L}, 1 \mu \mathrm{L})$ reverse transcriptase enzyme. After the addition of enzymes, the reaction mixture was further incubated at $42^{\circ} \mathrm{C}$ for 55 minutes. Finally, the reaction was terminated by incubation at $95^{\circ} \mathrm{C}$ for 7 minutes.

2.7. qPCR Application. To determine the mRNA expression levels of different cellular genes, real-time PCR was performed. The reaction mixture $(20 \mu \mathrm{l})$ consisted of $2 \mathrm{X}$ SYBR Green PCR Master Mix $(10 \mu$ l, Thermo Fisher Scientific, UK), template cDNA (100 ng), and forward $(0.2 \mu \mathrm{l}, 20 \mathrm{pmol})$ and reverse $(0.2 \mu \mathrm{l}, 20 \mathrm{pmol})$ primer. The real-time PCR was performed on a real-time PCR system (Applied Biosystem 7500/7500 Fast, USA). Preset two-step cycling profile consisted of $95^{\circ} \mathrm{C}$ (initial melting) for 10 minutes, followed by 40 cycles of $95^{\circ} \mathrm{C}$ (melting) for 15 seconds and $60^{\circ} \mathrm{C}$ (annealing and amplification) for 1 minute used for the amplification reaction. The obtained data were analyzed using the software $\left(\mathrm{CFX}\right.$ Maestro $\left.{ }^{\mathrm{TM}}\right)$ provided by the manufacturer.

\section{Results}

3.1. Characterized Quinolone-Resistant Klebsiella pneumoniae. The K. pneumoniae isolates $n=34$ (17\%) showed the biochemical API 20E (bioMérieux, France) profile including glucose fermentation, indole, $\mathrm{H}_{2} \mathrm{~S}$, and Methyl Red (MR) negative. All isolates were positive to Voges-Proskauer (VP). Additionally, all the positive isolates have hydrolyzed the urea. A PCR product of $1475 \mathrm{bp}$ against $16 \mathrm{~S}$ rDNA confirmed the molecular identification of K. pneumoniae (KY347730.1). Among the positive samples, $23(67 \%)$ isolates were found resistant to ciprofloxacin (Breakpoints; $\mathrm{MIC} \geq 4 \mu \mathrm{g} / \mathrm{ml})$. Both QRDR ( $g y r A$ and $g y r B)$, as well as PMQR ( $q n r A, q n r B$, and $q n r S)$, were detected in isolates that were found resistant to ciprofloxacin (Table 2).

In case of mutation, it was observed that gyrA showed a strong mutation (Ser83 $\longrightarrow$ Ile substitution) responsible for quinolone resistance. It was observed that amino acid present at 83-position is isoleucine (ATC) instead of serine (TCC). Accession numbers that were allotted to the isolates are MF953599 and MF953600.

3.2. Transcriptional Response of Klebsiella pneumoniae to Ciprofloxacin. In gene expression analysis, we found that the basal expression of DNA repair and protein folding elements under standard growth conditions were increased in the presence of ciprofloxacin (Figure 1). The mRNA levels of both mutS (DNA repair gene) and euTu (protein synthesis machinery) under the influence of ciprofloxacin were increased to many folds after 5 minutes of exposure and kept on increasing for up to 90 minutes (Figure 2). On $K$. pneumoniae exposure to ciprofloxacin, the mRNA levels of DNA damage response element ( $m u t S$ ) increased in a time-dependent fashion.

Moreover, we also found that ciprofloxacin toxicity modulated the expression pattern of chaperones-related genes $($ dnaK and $c l p B)$ and set off delayed cell divisions (Figure 2). We also observed that the expression of the dnaK gene constantly increased up to the initial 90 minutes; however, $c l p B$ expression declined after the initial 60 minutes. On the contrary, there was no significant change found in lexA gene expression (Figure 2). We quantify fts $Z$ gene expression and found that $K$. pneumoniae cell division was delayed due to ciprofloxacin-induced toxicity. Based on real-time PCR results, we observed that ciprofloxacin treatment altered the expression pattern of $f t s Z$ gene. In the presence of ciprofloxacin, the expression of $f t s Z$ was raised after 90 minutes which supports delayed cell division (Figure 3). Additionally, in current study, the gene expression results were also validated with spectrophotometric analysis, as shown in Figure 2.

\section{Discussion}

The emergence and evolution of multidrug-resistant bacterial strains are a complex issue that is significantly influenced by different factors such as the concentration of antibiotics. Some reports showed that sublethal concentrations of bactericidal antibiotics stimulated the development of MDR bacteria [16]. $K$. pneumoniae is a leading pathogen responsible for severe infections in humans and animals such as pneumonia, softtissue infections, and urinary tract infections, and the application of the irrational antibiotic further aggravates its severity. Ciprofloxacin is used extensively in clinical settings of Pakistan. However, K. pneumoniae has the ability to resist this antibiotic. The mutation in QRDR, i.e., gyrA and parC genes, is a key player responsible for quinolone resistance. 
TABle 2: Minimum inhibitory concentration (MIC) and plasmidmediated quinolone resistance (PMQR) genes in Klebsiella pneumoniae isolates.

\begin{tabular}{|c|c|c|c|c|}
\hline \multirow{2}{*}{ Isolate } & \multicolumn{3}{|c|}{ PMQR gene } & \multirow{2}{*}{ MIC of ciprofloxacin $(\mu \mathrm{g} / \mathrm{mL})$} \\
\hline & $q n r \mathrm{~A}$ & $q n r \mathrm{~B}$ & $q n r S$ & \\
\hline Kp1 & - & + & - & 64 \\
\hline $\mathrm{Kp} 2$ & - & + & - & 256 \\
\hline Kp3 & - & - & + & 64 \\
\hline Kp4 & - & - & + & 32 \\
\hline Kp5 & + & - & - & 16 \\
\hline Kp6 & - & + & + & 128 \\
\hline Kp7 & - & + & - & 64 \\
\hline Kp8 & - & - & + & 32 \\
\hline Kp9 & + & + & - & 128 \\
\hline Kp10 & - & + & + & 256 \\
\hline Kp11 & - & + & - & 128 \\
\hline Kp12 & - & - & + & 64 \\
\hline Kp13 & - & - & + & 64 \\
\hline Kp14 & - & - & + & 32 \\
\hline Kp15 & - & + & - & 128 \\
\hline Kp16 & - & - & + & 32 \\
\hline Kp17 & - & + & - & 64 \\
\hline Kp18 & - & + & - & 64 \\
\hline Kp19 & - & + & + & 128 \\
\hline Kp20 & - & + & - & 256 \\
\hline Kр21 & - & + & - & 256 \\
\hline Kp22 & - & + & - & 64 \\
\hline Kp23 & - & - & + & 32 \\
\hline
\end{tabular}

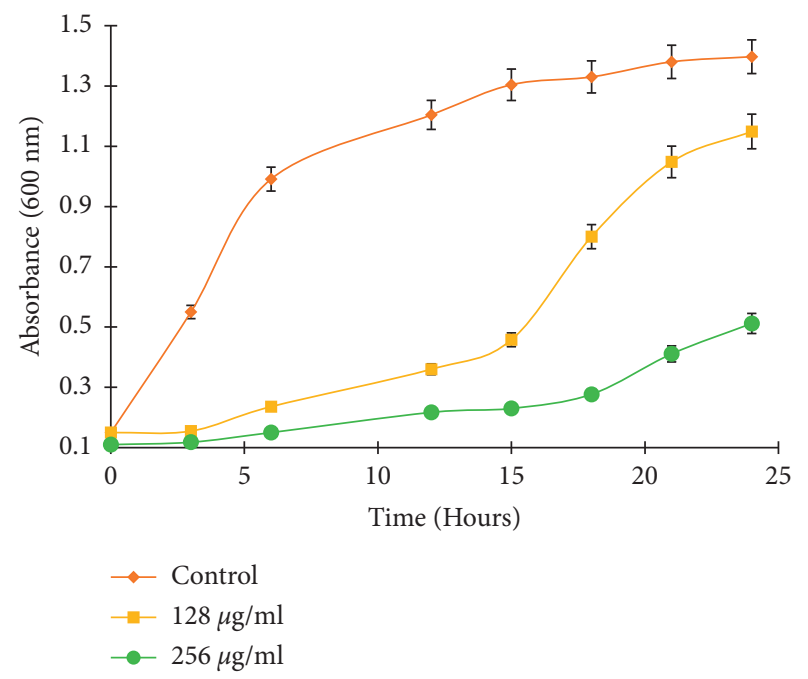

FIGURE 1: Growth curve of K. pneumoniae with ciprofloxacin treatment (conc. $128 \mu \mathrm{g} / \mathrm{ml}$ and $256 \mu \mathrm{g} / \mathrm{ml}$ ) and without ciprofloxacin treatment using standard culture conditions. The $X$-axis represents time (hours), whereas the $Y$-axis shows the absorbance $(\lambda=600 \mathrm{~nm})$.

Mutation in QRDR causes structural modifications in enzymes such as DNA gyrase/topoisomerase IV, which decreases the quinolone affinity $[17,18]$.

K. pneumoniae has also shown to produce persister cells by prolonged exposure to antibiotics [5]. According to our best knowledge, no such study has been reported from Pakistan which describes the transcriptional response in

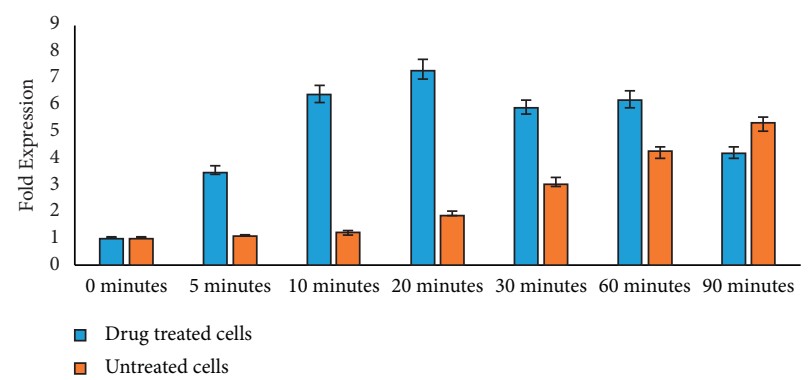

FIgURE 2: Transcriptomic profiling of Klebsiella pneumoniae clinical isolates under ciprofloxacin stress. The graph indicates alterations in the gene expression of euTu, dnaK, mutS, clpB, and lexA in a time-dependent manner. The $X$-axis represents genes, whereas the $Y$-axis displays the fold expression.

K. pneumoniae against the irrational antibiotic application in the healthcare settings of Pakistan.

It has already been established that increasing concentration of the antibiotic decreased the ability of $K$. pneumoniae to become a persister, and secondly, the exposure time duration was also a significant factor [5]. The mechanism of persister formation in K. pneumoniae is not yet clearly understood, but it is also present in many bacteria. In E. coli, quinolone antibiotics regulated the SOS response through lexA in a concentration-dependent manner, which is associated with the development of E. coli persister cells $[19,20]$. This SOS response induction steers the upregulation of various proteins associated with DNA repair which save bacteria from antibiotics and support persister development. On the other hand, DNA damage is the main cause of bacterial death as the concentration of quinolone increased. With extended exposure to quinolone treatment, the persisted bacteria die due to the loss of equilibrium between DNA repair and DNA damage. Though the DNA repair and SOS response are usually conserved in different bacteria, the main phenomenon of SOS response in K. pneumoniae still needs to be explored completely. In E. coli, the mechanism of persister formation has been elucidated [9]; if this phenomenon is the same in the case of K. pneumoniae, then it will be rational to hypothesize that DNA repair possesses a significant role in the formation of $K$. pneumoniae persister associated with ciprofloxacin concentration. Moreover, in the present study, delayed cell division of $K$. pneumoniae was observed in the case of ciprofloxacin treatment, and these findings corroborated the already established stress response elicited in a bacterial cell against antibiotic treatment [8]. Bacteria may exhibit some additional stress responses against antibiotics which may include hyperosmolarity, growth inhibition stress responses, nonoptimal growth temperature, $\mathrm{pH}$ downshift, and nutrient starvation [21].

In conclusion, in this study, we found that ciprofloxacin which is the most frequently used antibiotic in clinical settings of Pakistan induces various stress responses in indigenous $K$. pneumoniae isolates in a time- and concentration-depended on manner. Irrational and irregular prescription of this antibiotic may steer the formation of persister $K$. pneumoniae in the community which may ultimately be a serious threat to public health. 


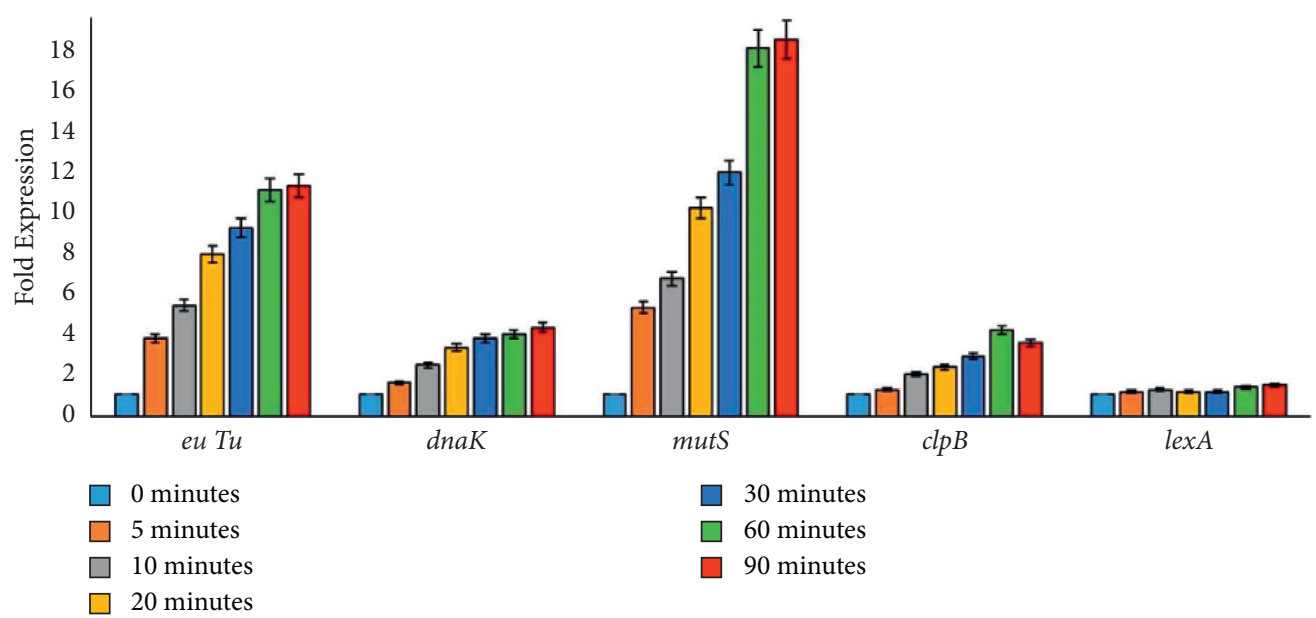

FIGURE 3: Transcriptomic profiling of Klebsiella pneumoniae clinical isolates under ciprofloxacin stress. The graph indicates a change in ftsZ expression in a time-dependent manner. The $X$-axis represents time, whereas the $Y$-axis displays the fold expression.

\section{Data Availability}

The aggregate data supporting the findings contained within this manuscript will be shared upon request submitted to the corresponding author. Identifying patient data will not be shared.

\section{Ethical Approval}

The protocol used in this study was in accordance with the Declaration of Helsinki and was approved by the Ethics Committee at Government College University, Faisalabad, Pakistan.

\section{Consent}

Written consent was individually obtained from each participant.

\section{Conflicts of Interest}

The authors have no conflicts of interest to declare.

\section{Acknowledgments}

The researchers would like to thank the Deanship of Scientific Research, Qassim University, for funding the publication of this project.

\section{References}

[1] A. Brauner, O. Fridman, O. Gefen, and N. Q. Balaban, "Distinguishing between resistance, tolerance and persistence to antibiotic treatment," Nature Reviews Microbiology, vol. 14, no. 5, pp. 320-330, 2016.

[2] E. Peterson and P. Kaur, "Antibiotic resistance mechanisms in bacteria: relationships between resistance determinants of antibiotic producers, environmental bacteria, and clinical pathogens," Frontiers in Microbiology, vol. 9, p. 2928, 2018.

[3] S. Navon-Venezia, K. Kondratyeva, and A. Carattoli, "Klebsiella pneumoniae: a major worldwide source and shuttle for antibiotic resistance," FEMS Microbiology Reviews, vol. 41, no. 3, pp. 252-275, 2017.

[4] F. Prestinaci, P. Pezzotti, and A. Pantosti, "Antimicrobial resistance: a global multifaceted phenomenon," Pathogens and Global Health, vol. 109, no. 7, pp. 309-318, 2015.

[5] H. Ren, X. He, X. Zou, G. Wang, S. Li, and Y. Wu, "Gradual increase in antibiotic concentration affects persistence of Klebsiella pneumoniae," Journal of Antimicrobial Chemotherapy, vol. 70, no. 12, pp. 3267-3272, 2015.

[6] A. G. Tkachenko, "Stress responses of bacterial cells as mechanism of development of antibiotic tolerance (review)," Applied Biochemistry and Microbiology, vol. 54, no. 2, pp. 108-127, 2018.

[7] J. L. Martinez, A. Fajardo, L. Garmendia et al., "A global view of antibiotic resistance," FEMS Microbiology Reviews, vol. 33, no. 1, pp. 44-65, 2009.

[8] K. Poole, "Stress responses as determinants of antimicrobial resistance in gram-negative bacteria," Trends in Microbiology, vol. 20, no. 5, pp. 227-234, 2012.

[9] M. Viveiros, M. Dupont, L. Rodrigues et al., "Antibiotic stress, genetic response and altered permeability of E. coli," PLoS One, vol. 2, no. 4, Article ID e365, 2007.

[10] J. A. Wiles, B. J. Bradbury, and M. J. Pucci, "New quinolone antibiotics: a survey of the literature from 2005 to 2010," Expert Opinion on Therapeutic Patents, vol. 20, no. 10, pp. 1295-1319, 2010.

[11] R. F. Alvi, B. Aslam, N. Shahzad, M. H. Rasool, and M. Shafique, "Molecular basis of quinolone resistance in clinical isolates of Klebsiella pneumoniae from Pakistan," Pakistan Journal of Pharmaceutical Sciences, vol. 31, no. 4, pp. 1591-1596, 2018.

[12] S. H. Ahmed, E. A. Daef, M. S. Badary, M. A. Mahmoud, and A. A. Abd-Elsayed, "Nosocomial blood stream infection in intensive care units at Assiut university hospitals (upper Egypt) with special reference to extended spectrum $\beta$-lactamase producing organisms," BMC Research Notes, vol. 2, no. 1, p. 76, 2009.

[13] CLSI, Performance Standards for Antimicrobial Susceptibility Testing, Clinical Laboratory Standards Institute, Wayne, PA, USA, Twenty-Eight edition, 2018.

[14] G. Jacoby, V. Cattoir, D. Hooper et al., "Qnr gene nomenclature," Antimicrobial Agents and Chemotherapy, vol. 52, no. 7, pp. 2297-2299, 2008. 
[15] R. Rosenblum, E. Khan, G. Gonzalez, R. Hasan, and T. Schneiders, "Genetic regulation of the ramA locus and its expression in clinical isolates of Klebsiella pneumoniae," International Journal of Antimicrobial Agents, vol. 38, no. 1, pp. 39-45, 2011.

[16] K. M. Jørgensen, T. Wassermann, P. Ø Jensen et al., "Sublethal ciprofloxacin treatment leads to rapid development of highlevel ciprofloxacin resistance during long-term experimental evolution of Pseudomonas aeruginosa," Antimicrobial Agents and Chemotherapy, vol. 57, no. 9, pp. 4215-4221, 2013.

[17] D. C. Hooper and G. A. Jacoby, "Topoisomerase inhibitors: fluoroquinolone mechanisms of action and resistance," Cold Spring Harbor Perspectives in Medicine, vol. 6, no. 9, 2016.

[18] M. Saeed, M. H. Rasool, F. Rasheed et al., "Extended-spectrum beta-lactamases producing extensively drug-resistant Salmonella Typhi in Punjab, Pakistan," Journal of Infection in Developing Countries, vol. 14, no. 2, pp. 169-176, 2020.

[19] T. Dörr, M. Vulić, and K. Lewis, "Ciprofloxacin causes persister formation by inducing the TisB toxin in Escherichia coli," PLoS Biology, vol. 8, no. 2, Article ID e1000317, 2010.

[20] N. Wu, L. He, P. Cui et al., "Ranking of persister genes in the same Escherichia coli genetic background demonstrates varying importance of individual persister genes in tolerance to different antibiotics," Frontiers in Microbiology, vol. 6, p. 1003, 2015.

[21] R. Hengge-Aronis, "Signal transduction and regulatory mechanisms involved in control of the $\sigma \mathrm{S}$ (RpoS) subunit of RNA polymerase," Microbiology and Molecular Biology Reviews, vol. 66, no. 3, pp. 373-395, 2002. 